\title{
Reliable Multicast Routing with Local Recovery Approach in Ad Hoc Network
}

\author{
Mehdi EffatParvar ${ }^{1}$, Mehdi Dehghan ${ }^{2}$, Ali Movaghar ${ }^{3}$, \\ Amir Dareshorzadeh $^{4}$ and MohammadReza EffatParvar ${ }^{5}$ \\ ${ }^{1}$ Islamic Azad University of Qazvin, Qazvin, Iran \\ ${ }^{2}$ Computer \& IT Eng Department of AmirKabir University of Technology, Tehran, Iran \\ ${ }^{3}$ Computer \& IT Eng Department of Sharif University of Technology, Tehran, Iran \\ ${ }^{4}$ Computer \& IT Eng Department of Iran University of Science and Technology, Tehran, Iran \\ ${ }^{5}$ Young Researcher Club, Islamic Azad University of Qazvin, Qazvin, Iran \\ E-mail:\{'effatparvar@qazviniau.ac.ir, ${ }^{2}$ dehghan@cic.aut.ac.ir, ${ }^{3}$ movaghar@sharif.edu\}
}

\begin{abstract}
An ad hoc network is a multi-hop wireless network formed by a collection of mobile nodes without the intervention of fixed infrastructure. Reliable Multicast plays a significant role in many applications of Mobile Ad Hoc Networks (MANETs). In recent years, a number of protocols have been proposed to deliver multicast packets reliably. In this paper we intend to propose a reliable multicast algorithm with local recovery approach, because importance of on-demand routing protocol we use this method in our algorithm. By using this algorithm nodes can join to multicast group in minimum time and data delivery will be increase. This method achieves fast recovery when route breakage happens, so destination can connect to source in new route or the same route.
\end{abstract}

\section{Introduction}

Mobile ad hoc network (MANET) is an economic solution for wireless communications, because it doesn't require any prior investment in fixed infrastructure. At the same time, the popularity of group computing has grown rapidly. Multicast is a very useful and efficient means of supporting group-oriented applications, especially in mobile/wireless environments where bandwidth is scarce and equipment has limited power. With the rapid growth of demand, the multicast technology in mobile ad hoc network (MANET) has attracted a lot of attention recently [1- 4].

When it became clear that group-oriented communication is one of the key application classes in MANET environments, a number of MANET multicast routing protocols have been proposed $[9,10]$. With maintaining routing state routing protocol classifies into two types: proactive and reactive. Proactive protocols maintain routing state, while the reactive reduce the impact of frequent topology changes by acquiring routes on demand. Previous study showed that reactive protocols performed better than proactive protocols.
Another criterion classifies protocols according to the global data structure used to forward multicast packets. Existing protocols are either tree- or mesh-based.

One of the main challenges for multicast routing in MANETs is the need to achieve robustness and reliability in the presence of universal mobility and frequent node outages. For this purpose, mesh-based protocols build a mesh for forwarding multicast data and thus robustness and reliability requirements with path redundancy inherent to meshes.

In addition to unicast routing protocols, several multicast routing protocols for ad hoc networks have been proposed in more recent years [8], [5], [11], [10], [12]. Unicast is a special form of multicast and some proposed multicast routing protocols support both unicast and multicast routing [8], [5].

[8], [10], [12] are generally more efficient in terms of data transmission than mesh-based protocols, but they are not robust against topology changes because there is no alternative path between a source and a destination. Meshbased multicast protocols [5], [11] provide alternative paths and a link failure need not trigger the recomputation of a mesh. Previous studies showed that mesh-based protocols are robust against topology change and are more suitable than tree-based protocols [11], [13]. ODMRP is an ad hoc multicast routing protocol based on a multicast mesh [5]. In ODMRP, if a source node has data to send, it periodically broadcasts "Join Request" to find and maintain multicast routes. All the other nodes re-broadcast the packet when they receive non-duplicate one. When a multicast group member receives "Join Request", the node replies with "Join Table." And subsequent replies by the nodes along a reverse path establish a route. ODMRP uses soft states, so leaving a group is automatically handled by timeout. As shown, ODMRP relies on frequent networkwide flooding, which may lead to a scalability problem when the number of source nodes is large. The control packet overhead becomes more prominent when the multicast group is small in comparison with the entire network. 
There are some reliable protocols in MANET; that we can classify them in three categories: The categories are, Automatic Retransmission Request (ARQ) based, gossipbased and Forward Error Correction (FEC) based. The protocols are RMA [2], RALM [1] and ReAct [14] are belong to the ARQ-based category.

In this paper we propose and evaluate our simulation result about reliability and scalability of ODMRP and then after analyzing its behavior, we proposed a new reliable algorithm with local recovery approach by using it and we call it LR-ODMRP; so by doing this method nodes can join to multicast group in minimum time and data delivery will be increase. This method achieves fast recovery when route breakage happens, so destination can connect to source in new route or the same route.

We have evaluated the performance of our algorithm via computer simulation. The simulation result shows that our algorithm effectively delivers data packets and is robust against frequent topology changes and in large number multicast group. In this method, data packet transmissions and control message exchanges are increased compared to ODMRP. We also observed that this method reduces the average packet delay.

The rest of paper is organized as follows. Section 2 presents the simulation results of reliability and scalability of ODMRP. In section 3 we proposed our algorithm with local recovery approach. Simulation result of this approach is reported in section 4. Section 5 follows with a conclusion and future work.

\section{Issue of Reliability and Scalability in ODMRP}

ODMRP [5] is a best-effort mesh-based multicast routing protocol. Hence, a major performance evaluation criterion is the average miss ratio, which is calculated as the number of packets that each receiver did not receive divided by the total number of packets sent by the source, and averaged over the total number of receivers in the multicast group. In this section, we study how the average miss ratio of ODMRP is affected by mobility, session size, and the transmission range. The simulation environment consists of 30 hosts in a $700 \mathrm{~m} * 700 \mathrm{~m}$ area. Three values of the transmission range of each host are considered: 200 m., $250 \mathrm{~m}$. and $300 \mathrm{~m}$. A single source multicasts 2000 512-byte DATA packet to a multicast group. Network bandwidth is $2 \mathrm{Mbit} / \mathrm{sec}$. MAC protocol is 802.11 . The underlying multicast routing protocol is ODMRP. The random waypoint [15] mobility pattern is used with equal minimum and maximum speeds of mobility (i.e., mobile hosts move with a constant speed). Several values of constant mobility speeds between $0 \mathrm{~m} / \mathrm{sec}$. (i.e., stationary hosts) and $25 \mathrm{~m} / \mathrm{sec}$. are considered. Figure 1 shows the miss ratio of ODMRP with different numbers of members.

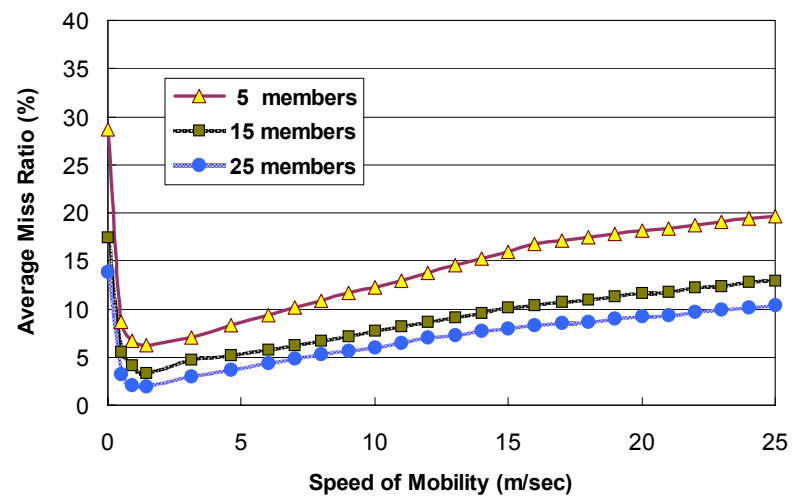

(a) Transmission range is $250 \mathrm{~m}$.

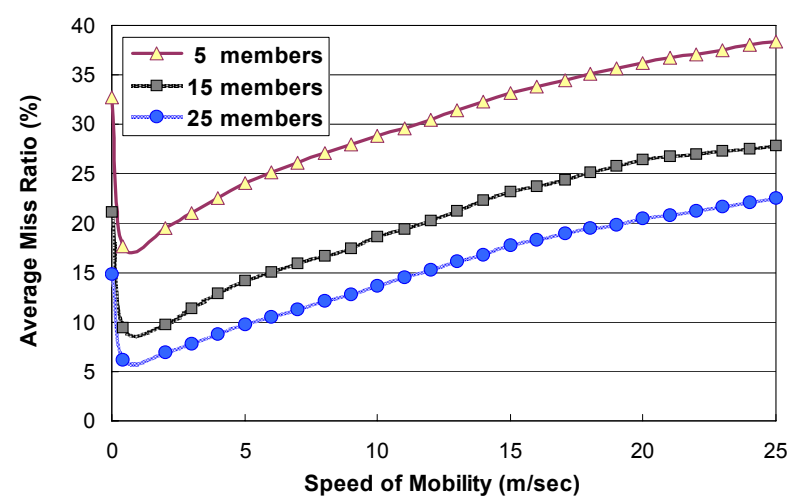

(b) Transmission range is $200 \mathrm{~m}$.

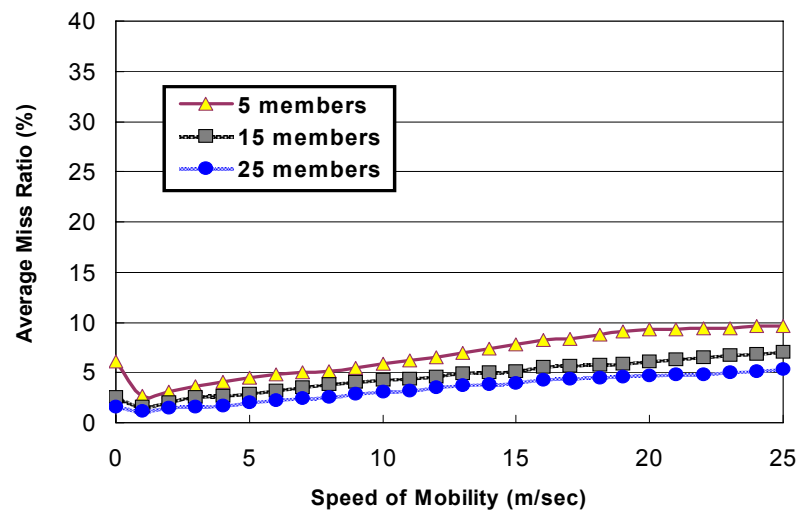

(c) Transmission range is $300 \mathrm{~m}$.

Figure 1: Average miss ratio versus speed of mobility for different numbers of members and transmission ranges

At zero speed of mobility, the hosts are stationary and thus, the topology of the ODMRP multicast mesh is fixed. Hence, when a source multicasts a data packet, only the 
receivers that are in the mesh may receive it but other receivers that are far away from the mesh will not. As mobility increases, distant receivers may, while moving, reach the multicast mesh and hence start receiving some data packets causing the average miss ratio to decrease (as shown in Figure 1). However, as mobility increases further, there are more frequent disconnections in the multicast mesh and hence packet loss increases.

For larger session sizes, there are more forwarding nodes in the multicast mesh, and therefore more redundancy in the mesh, leading to falling back on alternate multicast links when a multicast link fails and thus, reducing the average miss ratio.

ODMRP always tries to include the shortest path within the forwarding group, so almost it has lower latency than the other algorithm. In this section, Figures show the performance metrics as functions of group size. With fixed pause time as 60 seconds, we have one multicast group of size from 20 to 200. Figure (2) shows the result for packet delivery ratio. As group sizes increases, ODMRP delivers more fractions of packets. The reason is that the forwarding mesh becomes more reliable with more redundant paths as it increases its size.

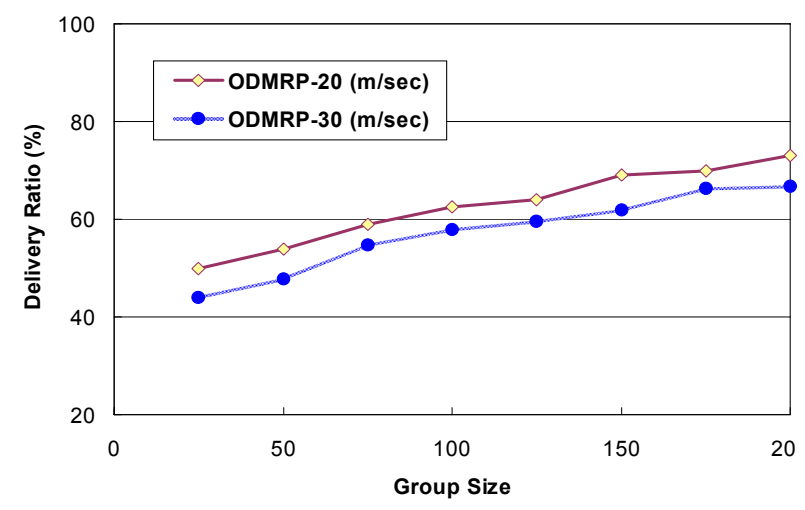

Figure 2: Packet delivery ratio versus group size. (Pause time is 60s, 1 group, 1 source, speed 20 and 30 $\mathbf{m} / \mathbf{s})$

In this section, we study the performance behaviors with respect to the horizontal scalability. We consider the following 6 scenarios: 72 by 2,48 by 3,36 by 4,24 by 6 , 18 by 8 and 12 by 12 . Here, "72 by 2 " means 2 multicast groups, and 72 members per group. Thus, in all scenarios, the total number of receivers is fixed to 144 . There is one source for each group. The traffic demand remains equal in all scenarios.

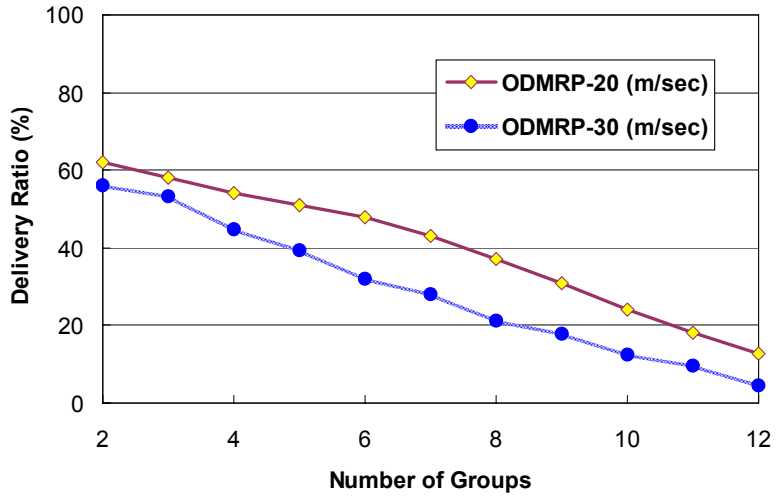

(a) Packet delivery ratio.

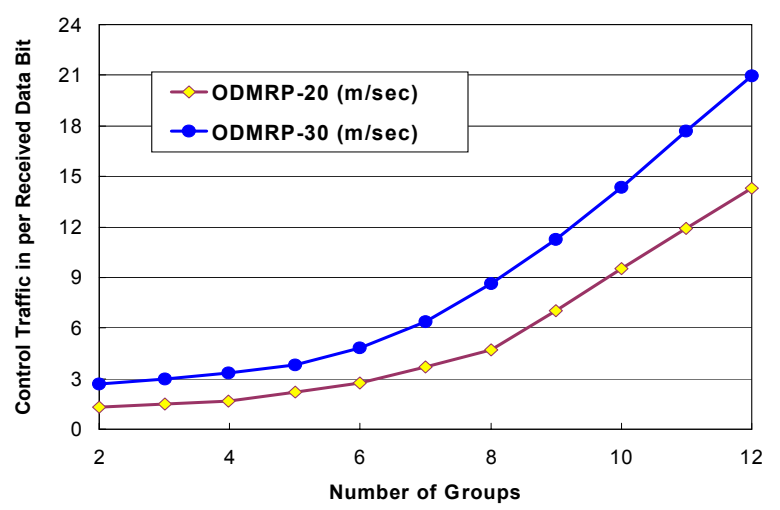

(b) Bit Overhead

Figure 3: Performance versus group size. (Pause time is 60s, 1 source per group, speed 20 and $30 \mathrm{~m} / \mathrm{s}$ )

Figure-3(a) shows the packet delivery ratio and the variance among the groups in the network. As the number of groups' increases, performance of ODMRP shows quick drop to less than $10 \%$ for 12 groups. The size of meshes does not decrease proportional to the group sizes.

Figure-3(b) shows the results for relative control bit overhead. The control traffic incurred by ODMRP increases dramatically with the increase in the number of groups and node speed. In ODMRP, after the source floods the JOIN QUERY message, all members should reply with JOIN REPLY packet. These reply packets will cause implosion problem when the group size is large. This problem is solved by aggregating the JOIN REPLY packets. When two JOIN REPLY packets reach one node, only one aggregated reply is needed to be forwarded further. However, with many groups of small size, the number of JOIN REPLY packets is huge and they have less chance to be aggregated. Thus, the control traffic 
increases significantly. The delivered packets are reduced, and this makes the value of relative control overhead increase even further. We can derive the following conclusions. When there are more multicast groups in the network, ODMRP has quick drop in all performance metrics.

\section{Reliable Multicasting and Local Recovery Mechanism}

After analyzing reliability and scalability on ODMRP, in this section we proposed a new recovery mechanism for ODMRP. Many routing algorithms use recovery method to increase the performance and decrease the delay between source and destination. Usually local recovery methods are used in tree based algorithm but sometimes because importance of data and our application we need to use this method in mesh based routing too. There is a number of works currently done on local route recovery, which include WAR (Witness-Aided Routing Protocol) [16], ABR (Associatively-Based Routing Protocol) and RDMAR (Relative Distance Micro discovery Ad-hoc Routing Protocol) [17]. In this section we introduce a new recovery approach while it keeping low complexity of the algorithm to a minimal. Complexity is an important attribute because nodes in MANET are generally small portable devices with little processing power, thus, the local recovery scheme must also cater to this property.

Because of many reasons in ODMRP it is possible that the discovered routes between source and destination break. Generally movement of node in MANET cause the route breakage,

also if this movement occurred by one FG node it is harmful than the other cases because by movement of FG node one of the important connecting route between this FG and all other node connected to that FG (e.g. destination or FG nodes) will break so they can not receive any packet until other route is discovered. So mostly we considered this case in our algorithm.

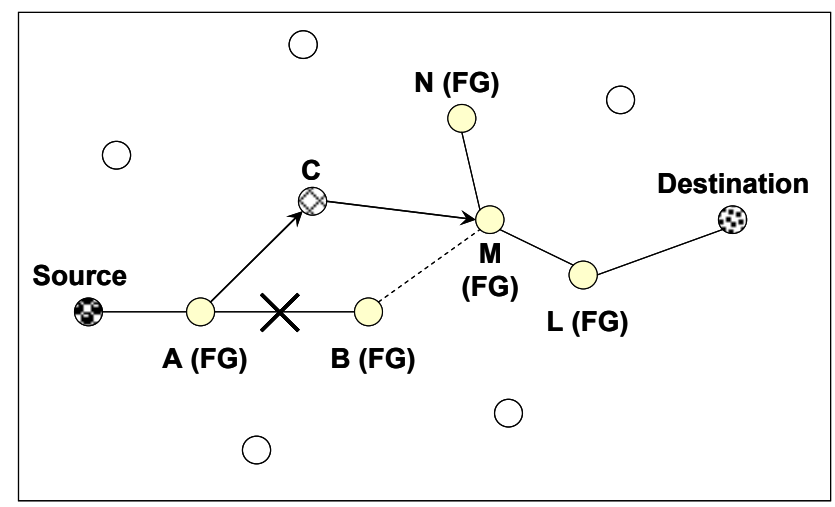

Figure 4: Local Recover approach in our algorithm

With reference to Figure 4, if the direct link (AB) breaks off, there should exist, in most cases, some indirect route from $\mathrm{A}$ to the original next node $\mathrm{B}$ through some neighbor node $\mathrm{C}$. In these situations, if a request packet is sent out to find the original next hop or other node which is at the further part of the original route with limited time-tolive (e.g. 2 hops), the possibility of repairing the current route should be high and the overhead should be much lower than using end-to-end global recovery. With the above assumptions, a new algorithm for the proposed this mechanism is described as follows:

When an intermediate FG node discovers that the link to the next hop has broken it would:

1. Save the data packet into local repair data buffer, set a timeout timer

2. Send out a two-hop wide local recovery request, containing a sequence of nodes including all the further nodes on the original route from $\mathrm{A}$ till the destination node D. Any node, on receiving the local recovery request, will check whether it is on the node list, and if it is, would send back a local recovery reply

3. On receiving local recovery request from an intermediate node, A salvages the data packet at the local repair buffer and transmits it using the repaired route.

4. If no reply received after the local repair timer expires, the data packet is dropped, and an error message is send back to the source from A, the source may then initiate a new round of end-to-end route discovery.

For doing the local recovering method we changed the Join-Reply packet in ODMRP, so each receiver generates a Join-Table packet that is broadcast to its neighbors. The Join-Table packet contains the multicast groups address sequence of (source address, next hop address) pairs and a count of the number of pairs. In one pair the source address and the next hop address is the same and it is equal with receiver address. When a node receives a Join-Table, it checks if the next node address of one of the entries matches its own address. If it does the node realize that it is on the path to the source and thus becomes a part of the forwarding group for that source by setting its forwarding group flag. It then adds its own Join-Table to last received Join-Table and broadcast it. By this method each FG node knows the other node on the path between itself and destination. So FG node can recover the breakage route in suitable time with minimal complexity and without need the source.

The simulator is implemented within the Glomosim library [15]. The Glomosim library is a scalable simulation environment for wireless network system using the parallel discrete event simulation capability provide by PARSEC [19]. In our simulation 50 nodes are randomly placed in $1000 \mathrm{~m}^{2}$ field. Default radio propagation range for each node is 225 meters; Nodes' channel bandwidth is set to 2 $\mathrm{Mbit} / \mathrm{sec}$. 
All member nodes join at the start of the simulation and remain members throughout the duration of the simulation. The source sends data at the rate of 20 packet/second. The size of data payload is 256 byte; random waypoint is used as the mobility model. Traffic model is constant bit rate (CBR). We consider some metric like packet delivery ratio, group reliability and routing overhead.

Mac protocol is 802.11 and transport protocol is UDP. Figure 5 show the protocol reliability varies with mobility (node speed).

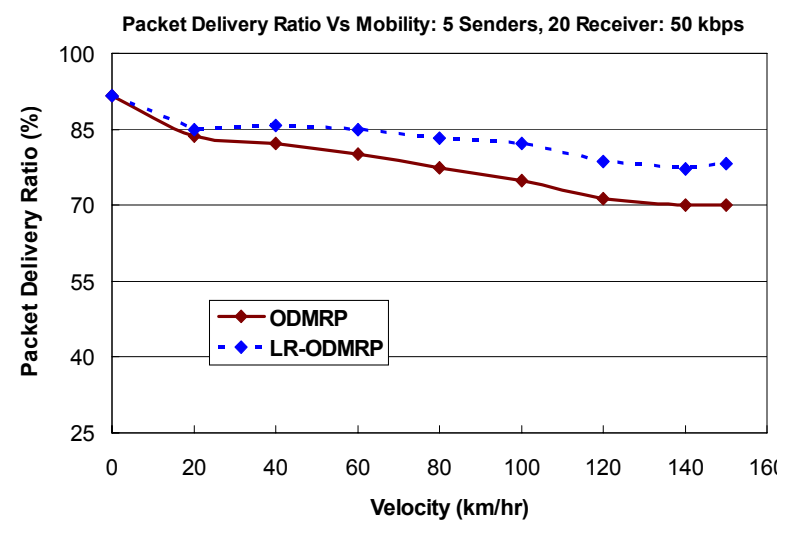

Figure 5: Packet delivery ratio as a function of node mobility

This comparing shows that at lower speed the difference in packet delivery ratio is not saleint.

In both case increased mobility requires that forwarding group member be updated more frequently also by increasing mobility causes frequent link change so by recovery method we can increase the packet delivery ratio. Figure 6 plots control overhead per data byte transferred as a function of mobility. Because of recovery packet in LRODMRP, this method slight increase overhead at higher speed.

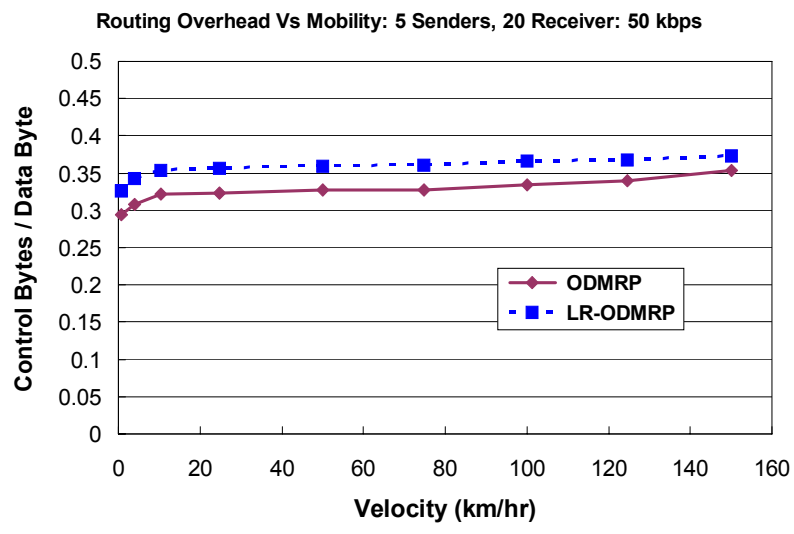

Figure 6: Routing overhead as a function of node mobility
Figure 7 plots group reliability as address function of node speed group reliability metric tries to capture the effectiveness of routing protocol in delivering packets to all group members.

ODMRP has poor performance even at low mobility. LR-ODMRP act like ODMRP but it can recover the link breakage and data packet drops.

Group Reliability Vs Mobility: 5 Senders, 20 Receiver: 50 kbps

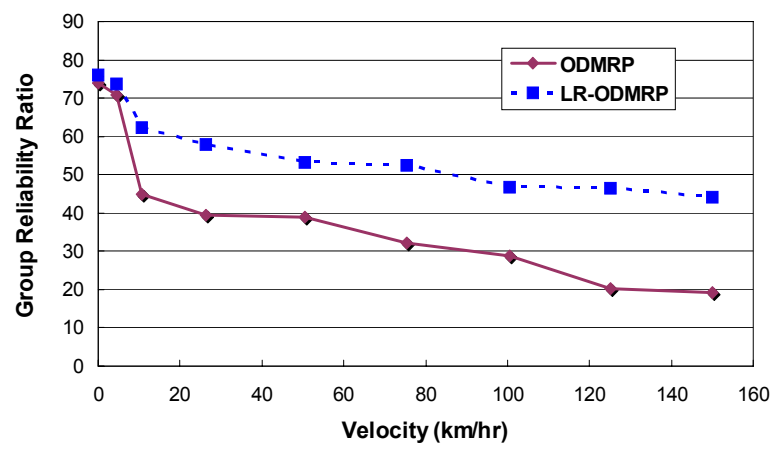

Figure 7: Group reliability as a function of node mobility

\section{Conclusion and Future Works}

An ad hoc network is a multi-hop wireless network formed by a collection of mobile nodes without the intervention of fixed infrastructure. Limited bandwidth and a high degree of mobility require that routing protocols for ad hoc networks be robust, simple, and energy conserving. On-demand routing protocol is an important category of the current ad-hoc routing protocols, in which a route between a communicating node pair is discovered only on demand. The On-Demand Multicast Routing Protocol (ODMRP) falls into the reactive protocol category since group membership and multicast routes are established and updated by the source whenever it has data to send. ODMRP has simple structure so we use this protocol to design reliable multicast routing with local recovery approach with minimal complexity. In this paper, we study the reliability and scalability of ODMRP and our algorithm. Simulation results have shown that in ODMRP the average miss ratio decreases with increasing the number of multicast group members, which indicates that ODMRP has more packet delivery capabilities for denser multicast groups. In addition, our simulations have also shown that using ODMRP, the average miss ratio does not always increase with increasing the speeds of mobility of the mobile hosts. Instead, there is a range of mobility speeds in which the average miss ratio decreases with increasing speeds of mobility and after that range, the average miss ratio increases with increasing speeds of mobility. We have presented a new scalable local recovery mechanism. Our simulation showed that LR-ODMRP is more reliable than the ODMRP, also it has good 
performance when we increase the speeds of mobility and multicast group member, Control packet in ODMRP is less than our algorithm but we showed that ODMRP is not a scalable when we increase the number of multicast group. We can use LR-ODMRP in large scale topology and we can increase the packet delivery ratio and reliability.

For the future work we intend to use clustering algorithm and we proposed a new reliable algorithm with scalability advantage, also we can develop our recovery approach to use in other multicast routing.

\section{References}

[1] K. Tang, K. Obraczka, S.-J. Lee, M. Gerla, "A reliable, congestion-control led multicast transport protocol in multimedia multi-hop networks," The 5th International Symposium on Wireless Personal Multimedia Communications, Volume: 1, Pages:252-256 , 27-30 Oct. 2002.

[2] T. Gopalsamy, M. Singhal, D.Panda, and P. Sadayappan, "A reliable multicast algorithm for mobile ad hoc networks", In Proceedings of ICDCS, 2002, July 02-05, 2002.

[3] J. Luo, P.T. Eugster, J.-P. Hubaux, "Route driven gossip: probabilistic reliable multicast in ad hoc networks," INFOCOM 2003, Volume: 3, Pages:2229-2239, 30 March-3 April 2003.

[4] R. Chandra, V. Ramasubramanian, K. Birman, "Anonymous Gossip: improving multicast reliability in mobile ad-hoc networks," The 21st International Conference on Distributed Computing Systems, Pages:275-283, 16-19 April 2001.

[5] M. Gerla, S.-J. Lee, W. Su., "On-demand multicast routing protocol (ODMRP) for ad hoc networks," IETF Internet Draft, draft-ietf-manet-odmrp-02.txt, 2000.

[6] J. Garcia-Luna-Aceves and E. Madruga. A multicast routing protocol for ad-hoc networks. In Proc. of INFOCOM'99, pages $784\{792$, March 1999.

[7] P. Jacquet, P. Minet, A. Laouiti, L. Viennot, T. Clausen, and C. Adjih. Multicast optimized link state routing. IETF manet draft-ietf-manet-olsr-molsr-01.txt, 2002.

[8] E. Royer and C. Perkins. Multicast operation of the ad-hoc on-demand distance vector routing protocol. Proceedings of the ACM Mobicom '99, pages 207 \{218, August 1999.
[9] P. Sinha, R. Sivakumar, and V. Bharghavan. MCEDAR: Multicast core extraction distributed ad-hoc routing. In Proc. of the Wireless Communications and Networking Conference, 1999.

[10] C. Wu, Y. Tay, and C. Toh. Ad hoc Multicast Routing protocol utilizing Increasing id-numberS (AMRIS). IETF manet (draft-ietf-manet-amris-spec-00.txt), 1998.

[11] J. J. Garcia-Luna-Aceves, and E. L. Madruga, "The coreassisted mesh protocol," IEEE Journal on Selected Area in Communications, Special Issue on Ad-Hoc Networks, Vol. 17, No. 8, Aug. 1999.

[12] E. Bommaiah, M. Lui, A. McAuley, and R. Talpade, "AMRoute: adhoc multicast routing protocol," Internet draft, IETF, Aug. 1998.

[13] S. Lee, W. Su, J. Hsu, M. Gerla, and R. Bagrodia, "A performance comparison study of ad hoc wireless multicast protocols," INFOCOM 2000, Mar. 2000.

[14] V. Rajendran, Y. Yi, K. Obraczka, S.J.Lee, K.Tang and M. Gerla, "Reliable, Adaptive, Congestion-Controlled Adhoc Multicast Transport Protocol: Combining Source-based and Local Recovery", UCSC Technical Report, 2003.

[15] X. Zeng, R. Bagrodia, and M. Gerla, "GloMoSim: A Library for Parallel Simulation of Large-scale Wireless Networks," Proc. of the 12th Workshop on Parallel and Distributed Simulation, Banff, Alberta, Canada, July 1998, pp. 154-161.

[16] Aron and S. Gupta. "A Witness-Aided Routing Protocol for Mobile Ad Hoc Networks with Unidirectional Links", Proc. First Int Conf. on Mobile Data Access (MDA '99), Hong-Kong, Dec. 1999, pp. 24-33.

[17] G. Aggelou , R. Tafazolli, "RDMAR: a bandwidth-efficient routing protocol for mobile ad hoc networks", Proceedings of the 2nd ACM international workshop on Wireless mobile multimedia, Seattle, Washington, United States, August 20-20, 1999, pp.26-33.

[18] UCLA Parallel Computing Laboratory and Wireless Adaptive Mobility Laboratory, GloMoSim: A Scalable Simulation Environment for Wireless and Wired Network Systems.

[19] R. Bagrodia, R. Meyer, M. Takai, Y. Chen, X. Zeng, J. Martin, and H.Y. Song, "PARSEC: A Parallel Simulation Environment for Complex Systems," IEEE Computer, vol. 31, no. 10, Oct. 1998, pp.77-85. 